\title{
THE CANADIAN JOURNAL OF
}

Neurological Sciences

\section{LE JOURNAL CANADIEN DES}

Sciences Neurologiques

AN INTERNATIONAL JOURNAL / UN JOURNAL INTERNATIONAL

1 Message from the Editor

\section{ORIGINAL ARTICLES}

3 Kinematics of Initiating a Two-Joint Arm Movement in Patients with Cerebellar Ataxia Steve Massaquoi and Mark Hallett

15 Reciprocal Inhibition in Hemiplegia: Correlation with Clinical Features and Recovery Yasuyuki Okuma and Robert G Lee

24 Vagal Nerve Complex in Normal Development and Sudden Infant Death Syndrome LE Becker and W Zhang

34 Stereotactic Management of Bacterial Brain Abscesses Sohrab Shahzadi, Andres M Lozano, Mark Bernstein, Abhijit Guha and Ronald R Tasker

40 Acute Hydrocephalus Following Aneurysmal Subarachnoid Hemorrhage $V$ Mehta, RO Holness, $K$ Connolly, $S$ Walling and $R$ Hall

46 Tirilazad Prevention of Reperfusion Edema After Focal Ischemia in Cynomolgus Monkeys Donald P Boisvert and Edward D Hall

53 Symptomatic Dystonias Associated with Structural Brain Lesions: Report of 16 Cases Vladimir S Kostic, Marina Stojanovic-Svetel and Aleksandra Kacar

57 Prior Intra-operative Hypotension is not a Risk Factor for Development of Alzheimer's Disease NI Bohnen, EFM Wijdicks, E Kokmen, MA Warner and LT Kurland

59 Narcolepsy Secondary to Fourth Ventricular Subependymoma TFK Ma, LC Ang, M Mamelak, SJ Kish, B Young and AJ Lewis

\section{HISTORICAL NEUROLOGY AND NEUROSURGERY}

63 A History of Neurology in Toronto 1892-1960: Part II John $R$ Wherrett

31st CANADIAN CONGRESS OF NEUROLOGICAL SCIENCES

June $25-29,1996$ London, Ontario

76 On the Names of Babiński Andrew P Gasecki and Vladimir Hachinski

80 George A Savoy, Visionary Benefactor of Canadians with Epilepsy, and the History of the Savoy Foundation for Epilepsy CM Rémillard, BG Zifkin, A Sherwin and W Feindel

The official Journal of: The Canadian Neurological Society, The Canadian Neurosurgical Society, The Canadian Society of Clinical Neurophysiologists, The Canadian Association for Child Neurology 
With Epival, it can be.

Because Epival has been proven effective in primary generalized epilepsy, ${ }^{1 \cdot 3}$ as well as in partial seizures that secondarily generalize. ${ }^{4,5^{*+}}$

Epival has been associated with little effect on learning and cognition. ${ }^{6}$

Drowsiness, visual disturbances, and ataxia are rarely noted ${ }^{7}$ - unlike phenytoin and carbamazepine. ${ }^{8}$ Epival is generally well tolerated in properly screened patients, ${ }^{7}$ causing less

GI irritation (nausea, vomiting and indigestion) than valproic acid. ${ }^{9}$

With Epival, your epilepsy patients can be confident that they most likely appear to be just

like anyone else. Because there's more to anticonvulsant therapy than seizure control.

\section{THIS SHOULD BE THE ONLY INDICATION THEY HAVE EPILEPSY.}
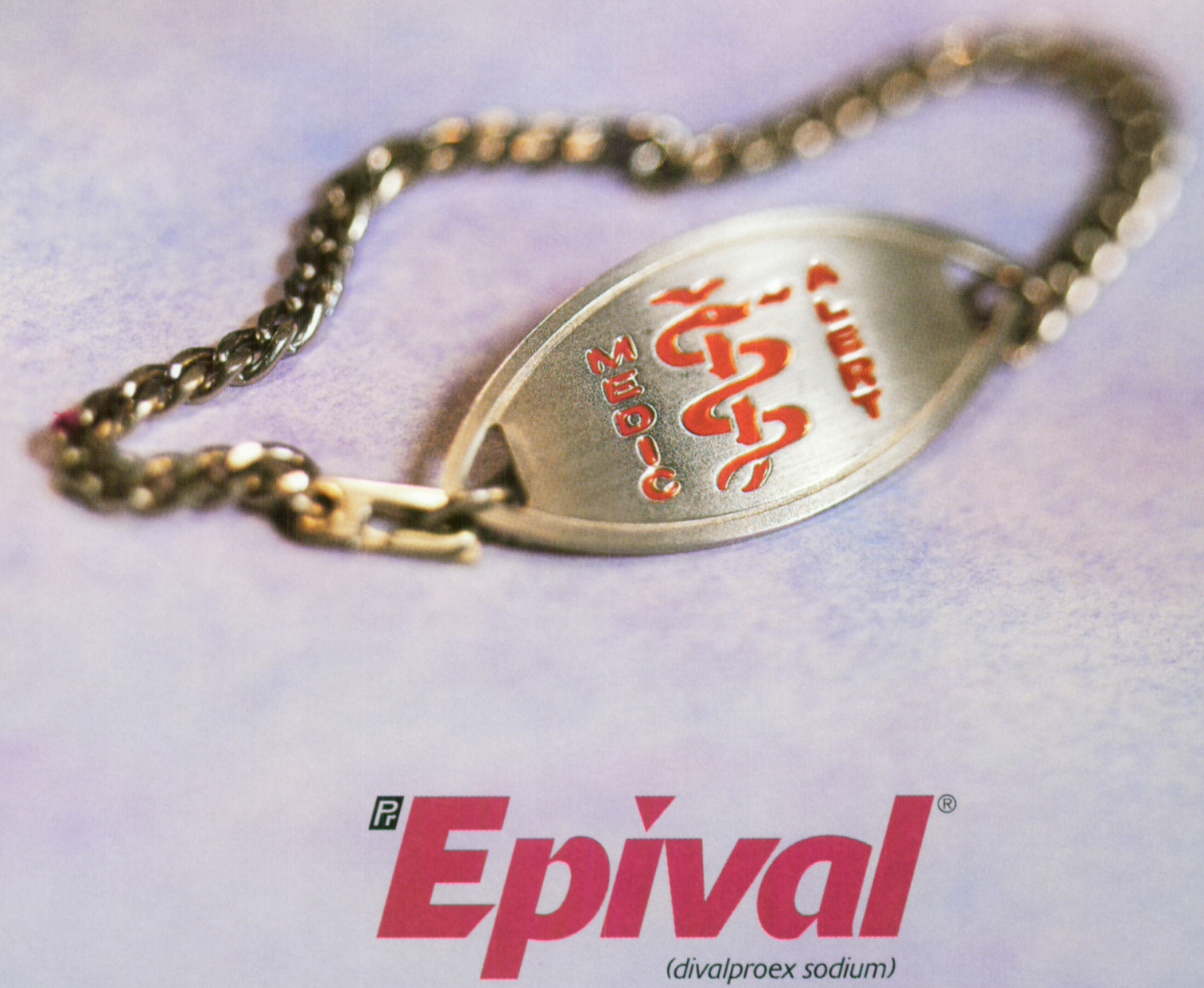

HELPS PUT PATIENTS BACK IN CONTROL. 


\section{THE CANADIAN JOURNAL OF}

\section{Neurological Sciences}

\section{LE JOURNAL CANADIEN DES}

\section{Sciences Neurologiques}

ORIGINAL ARTICLES

1 Message from the Editor

3 Kinematics of Initiating a Two-Joint Arm Movement in Patients with Cerebellar Ataxia Steve Massaquoi and Mark Hallett

15 Reciprocal Inhibition in Hemiplegia: Correlation with Clinical Features and Recovery Yasuyuki Okuma and Robert G Lee

24 Vagal Nerve Complex in Normal Development and Sudden Infant Death Syndrome LE Becker and W Zhang

34 Stereotactic Management of Bacterial Brain Abscesses Sohrab Shahzadi, Andres M Lozano, Mark Bernstein, Abhijit Guha and Ronald R Tasker

40 Acute Hydrocephalus Following Aneurysmal Subarachnoid Hemorrhage $\checkmark$ Mehta, RO Holness, K Connolly, $S$ Walling and $R$ Hall

46 Tirilazad Prevention of Reperfusion Edema After Focal Ischemia in Cynomolgus Monkeys Donald P Boisvert and Edward D Hall

53 Symptomatic Dystonias Associated with Structural Brain Lesions: Report of 16 Cases Vladimir S Kostic, Marina Stojanovic-Svetel and Aleksandra Kacar

57 Prior Intra-operative Hypotension is not a Risk Factor for Development of Alzheimer's Disease NI Bohnen, EFM Wijdicks, E Kokmen, MA Warner and LT Kurland

59 Narcolepsy Secondary to Fourth Ventricular Subependymoma TFK Ma, LC Ang, M Mamelak, SJ Kish, B Young and AJ Lewis

Historical NEUROLOGY AND NEUROSURGERY
63

A History of Neurology in Toronto 1892-1960: Part II

John $R$ Wherrett

76 On the Names of Babiński

Andrew P Gasecki and Vladimir Hachinski

80 George A Savoy, Visionary Benefactor of Canadians with Epilepsy, and the History of the Savoy Foundation for Epilepsy

CM Rémillard, BG Zifkin, A Sherwin and W Feindel

Books Received 83

Book Reviews 84

Notes and Announcements 89

Erratum 90

Calender of Events 91

Instructions to Authors xiv

Advertisers Index xxxi 
THE CANADIAN JOURNAL OF

Neurological Sciences

\section{LE JOURNAL CANADIEN DES \\ Sciences Neurologiques}

\section{Editor/Rédacteur en chef}

James A. Sharpe TORONTO, ON

Associate Editors/Rédacteurs associés

Laurence E. Becker TORONTO, ON

John P. Girvin LONDON, ON

John R. Wherrett TORONTO, ON

\section{Past Editors}

Robert G. Lee CALGARY, AB

Robert T. Ross WINNIPEG, MB

(founding editor)

\section{Editorial Board/Conseil Scientifique}

Jack P. Antel MONTREAL, QC

Warren T. Blume LONDON, ON

Jean-Pierre Bouchard QUÉBEC, QC

Peter R. Camfield HALIFAX, NS

Pierre Duquette MONTRÉAL, QC

Peter J. Dyck ROCHESTER, MN, USA

Andrew Eisen VANCOUVER, BC

Julian T. Hoff ANN ARBOR, MI, USA

Renn Holness HALIFAX, NS

Peter Humphreys OTTAWA, ON

George Karpati MONTRÉAL, QC

Patrick L. McGeer VANCOUVER, BC

John H. Noseworthy ROCHESTER, MN, USA

C. Warren Olanow NEW YORK, NY, USA

William Pryse-Phillips ST. JOHNS, NF

Ali H. Rajput SASKATOON, SK

James T. Rutka TORONTO, ON

Alan M. Smith MONTRÉAL, QC

Garnette R. Sutherland CALGARY, AB

Jean-Guy Villemure MONTRÉAL, QC

Douglas Zochodne CALGARY, AB

Book Review Editor / Rédacteur de critiques de livres

Mary Anne Lee CALGARY, AB

News Editor/Rédacteur (nouvelles)

John W. Norris TORONTO, ON

Managing Editor/Administratrice adjointe

Sally A. Gregg CALGARY, AB

Publications Committee/Comité de Rédaction

Frances Booth WINNIPEG, MB

Donald Brunet KINGSTON, ON

Mark Hamilton CALGARY, AB

William Pryse-Phillips ST. JOHN's, NF
The official journal of: / La Revue officielle de:

The Canadian Neurological Society

La Société Canadienne de Neurologie

The Canadian Neurosurgical Society

La Société Canadienne de Neurochirurgie

The Canadian Society of Clinical Neurophysiologists La Société Canadienne de Neurophysiologie Clinique

The Canadian Association of Child Neurology

L'Association Canadienne de Neurologie Pédiatrique

The permanent secretariat for the four societies and the Canadian Congress of Neurological Sciences is at/

Le secrétariat des quatre associations et du Congrès Canadien des Sciences Neurologiques est situe en permanence à:

810, 906 - 12 Avenue S.W., Calgary, AB Canada T2R IK7

The Canadian Journal of Neurological Sciences is published quarterly. The annual subseription rate is $\$ 65$ for members; $\$ 75$ for non-members in Canada: $\$ 85$ for USA and elsewhere. Residents. Interns. Pre- and Post-Doctoral Students $\$ 32.50$ per annum (members): $\$ 37.50$ per annum (non-members). Single copies $\$ 20$ each plus postage and handling. All manuscripss and conmunications should be sent to: Canadian Journal of Neurological Sciences, P.O. Box 4220, Station C. Calgary, AB Canada T2T 5N1. Courier to: $810.906-12$ th Avenue S.W.,

Calgary, AB Canada T2R IK7. Telephone (403) 229-9575: Fux (403) 229-1661 E-mail: cjns@canjneurolsci.org

COPYRIGHTO 1996 by THE CANADIAN JOURNAL OF NEUROLOGICAL SCIENCES INC. No part of this journal may be reproduced in any form without the prior pennission of The Canadian Joumal of Neurological Sciences. Mailed under Publications Mail registration number 3307. Postage paid at Calgary. Alberta. This journal is indexed by Index Medicus, Excerpta Medica and Currem Contents Clinical Practice and Life Sciences, Curremt Awareness in Biological Sciences.

Le Journal Canadien des Sciences Neurologiques est publié trimestriellement. Labonnement annuel est de $65 \$$ pour les membres; 75 pour les non-membres au Canada: 85 \$ pour les Ents Unis et ailleurs. Intemes, résidents, fellow's pré et post doctoral: $32.50 \$$ par année (membres); $37,50 \$$ par année (non-membres). Copie simple: $20 \$$ plus affranchissement et manutention. Toutes les communications et les manuscrits doivent être adressés a Journal Canadien des Sciences Neurologiques, P.O. Box 4220, Station C. Calgary. AB Canada T2T 5N1. Par courrier: 810,906 - 12th Avenue S.W.. Calgary, AB Canada T2R IK7.

Téléphone (403) 229-9575: Fax (403) 229-1661. E-mail çns@cunjneurolsci.org DROITS D AUTEURO 1996: THE CANADIAN JOURNAL OF

NEUROLOGICAL SCIENCES INC. Aucune partie de ce Joumal ne peut être reproduite, sous quelque forme que ce soit, sans la l'authorisation du Journal Cannadien des Sciences Neurologiques. Posté sous permis de poste-publications no 3307. Port payé a Calgary, Alberta. Le Journal est cité et indexé dans Inder Medicus, Excerpta Medica et Current Contents - Clinical Practice at Life Sciences, Current Awareness in Biological Sciences.

Advertising representative/Représentant de publicité: Sally Gregg, Canadian Journal of Neurological Sciences $810,906-12$ Ave. S.W., Calgary, AB Canada T2R 1K7 Tel (403) 229-9575 Fax (403) 229-1661

E-mail: cjns@canjneurolsci.org

Printer/lmprimeur:

McAra Printing Limited, 105, 2507 - 12th Street N.E., Calgary, Alberta T2E 7L5

ISSN $0317-167$ 


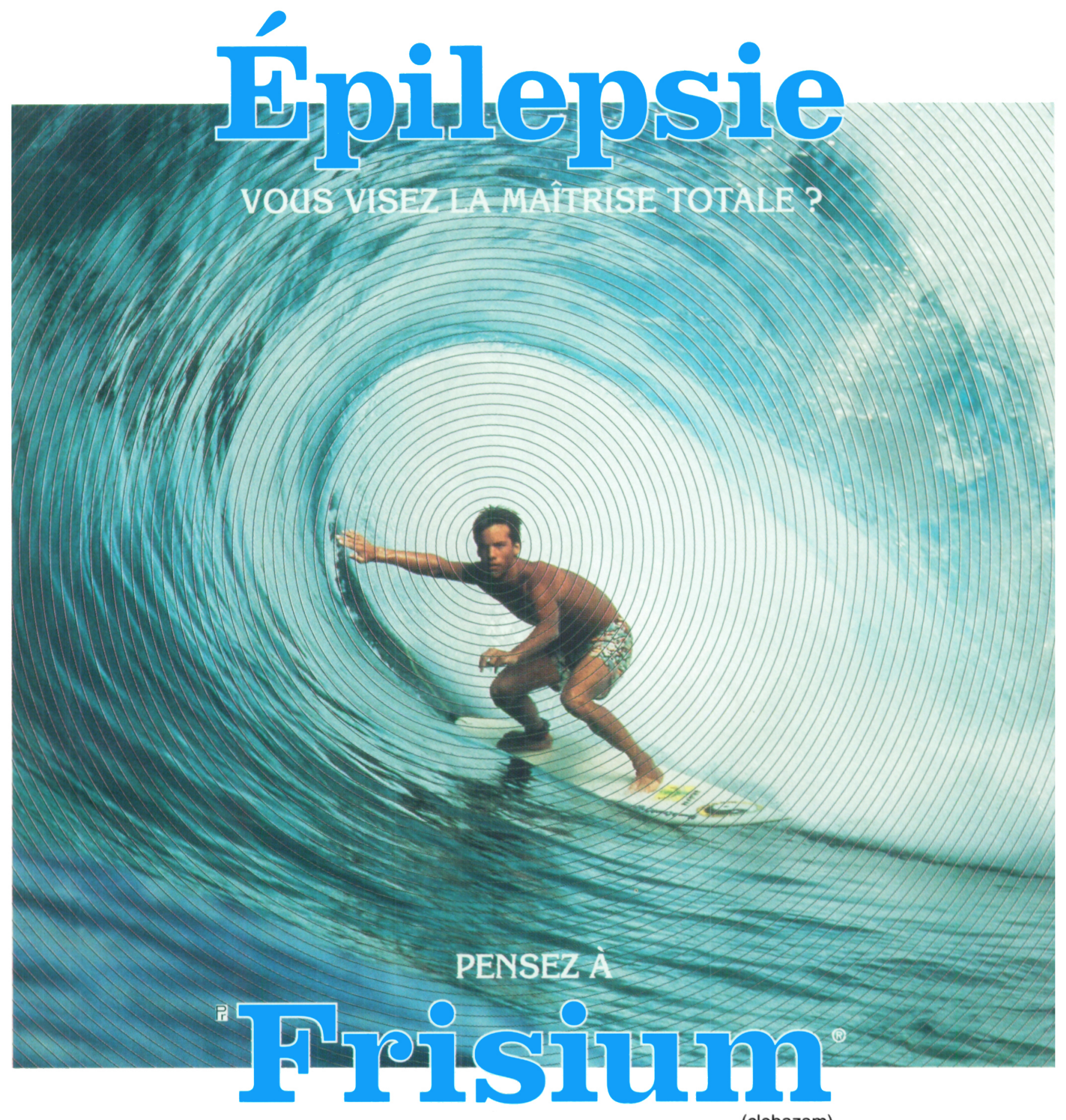

(clobazam)

- Maîtrise totale des crises chez un pourcentage impressionnant de patients ${ }^{1}$.

- Frisium est "un antiépileptique remarquablement efficace et [généralement] sûr lorsqu'il est ajouté au traitement ${ }^{1}$.
- Frisium est efficace contre tous les types de crises de l'enfant et de l'adulte ${ }^{2}$.

- La posologie est d'une dose par jour, à prendre de préférence au coucher. *

\section{Pour une approche globale de la maîtrise des crises}

*La dose quotidienne peut être fractionnée chez certains patients.

Frisium est indiqué pour le traitement adjuvant des épileptiques lorsqu'un traitement anticonvulsivant habituel ne suffit pas à stabiliser. Comme avec toutes les benzodiazépines, les patients, surtout les personnes âgées, doivent donc en être prévenus. Les effets indésirables les plus fréquents (>1\%) sont l'ataxie, le gain de poids, les étourdissements et la nervosité. 
New Lamictal-
Adjunctive Antiepileptic Therapy

\section{Control over a wide with a low CNS}

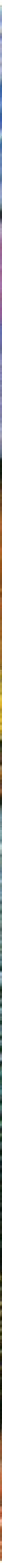

${ }^{t}$ Withdrawal rates $(\geq 0.6 \%$ ): dizziness $2.4 \%$, headache $1.3 \%$, nausea $1.3 \%$, blurred vision $1.1 \%$, rash $1.1 \%$, diplopia $0.7 \%$, ataxia $0.6 \%$. If there is any unexplained rash, fever flu-like symptoms or worsening of seizure control, then hepatic, renal and clotting parameters should be monitored. See Product Monograph for recommendations when prescribing for geriatric patients and for patients with impaired renal and/or liver function. Serious skin-related events may be related to rapid initial titration of dosing and

fAs with most other AEDs, before prescribing LAMICTAL, refer to Product Monograph for possible drug interactions with other AEDs.

Glaxo Wellcome 


\section{Sooner or later, every migra again. Imitrex ${ }^{\circ}$ believes}

A patient who complains about migraine is also complaining about a disrupted life. Indeed, research shows that in at least $31 \%$ of attacks, migraine sufferers cannot continue with their daily activities.

That's where Imitrex ${ }^{\circledast}$ comes in. For most
Unlike conventional remedies, it has not been shown to cause medication-induced headache ${ }^{3,6-8}$ Its adverse events are generally well tolerated, quickly resolved and usually non-threatening when explained to the patient." ${ }^{* 3,7,9}$ Imitrex ${ }^{\oplus}$ may be more expensive, but patients, Imitrex can bring

complete relief between 90

minutes and 2 hours, versus up to

9 hours for the usual treatments..$^{2,3}$

Imitrex treats all the symptoms

of migraine. ${ }^{* 35}$

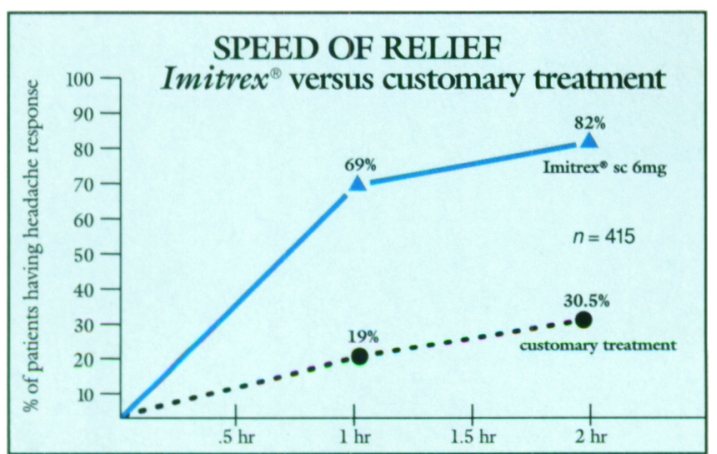

over 250,000 Canadian patients

continue to choose it for

migraine relief. ${ }^{10}$

The successful use of

Imitrex ${ }^{\circledast}$ is most likely in patients

who understand its common 


\section{ine sufferer will feel normal}

\section{it should be sooner.}

side effects, and who know when the drug should be used." ${ }^{* 11}$ Imitrex ${ }^{\circledast}$ should be taken at the start of a

debilitating attack, and may also be used after the

failure of conventional treatments (except ergotamine-

containing preparations) ${ }^{3}$
Most patients have attacks that limit normal function. ${ }^{1,12}$ So give your patients ${ }^{\dagger}$ the option of using Imitrex. It's a proven route to a fast recovery. ${ }^{2}$

For more information about Imitrex, please

call 1-800-268-0324.
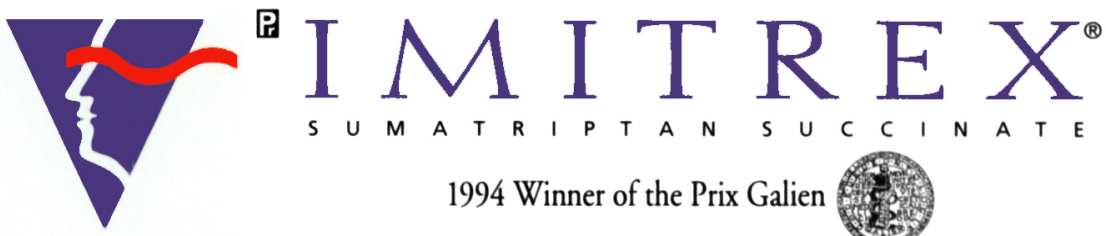

1994 Winner of the Prix Galien

\section{A faster way back.}

Glaxo

Glaxo Canada Inc.

*Customary treatments include simple analgesics, combination analgesics, ergot derivatives, NSAIDs, narcotics, antiemetics, others. 2 **Head pain, nausea, vomiting, photophobia and phonophobia. ${ }^{*}{ }^{* * * F a t i g u e}$ "Cizziness, nausea and vomiting have persist, patient should immediately consult physician. $3^{\dagger}$ Contraindicated in patients with ischaemic heart disease, angina pectoris including Prinzmetal angina, previous myocardial infarction and uncontrolled persist, patient should immediately consult physician. ${ }^{3}$ Contraindica 
IN $N$ T $O$ D U C O I N G
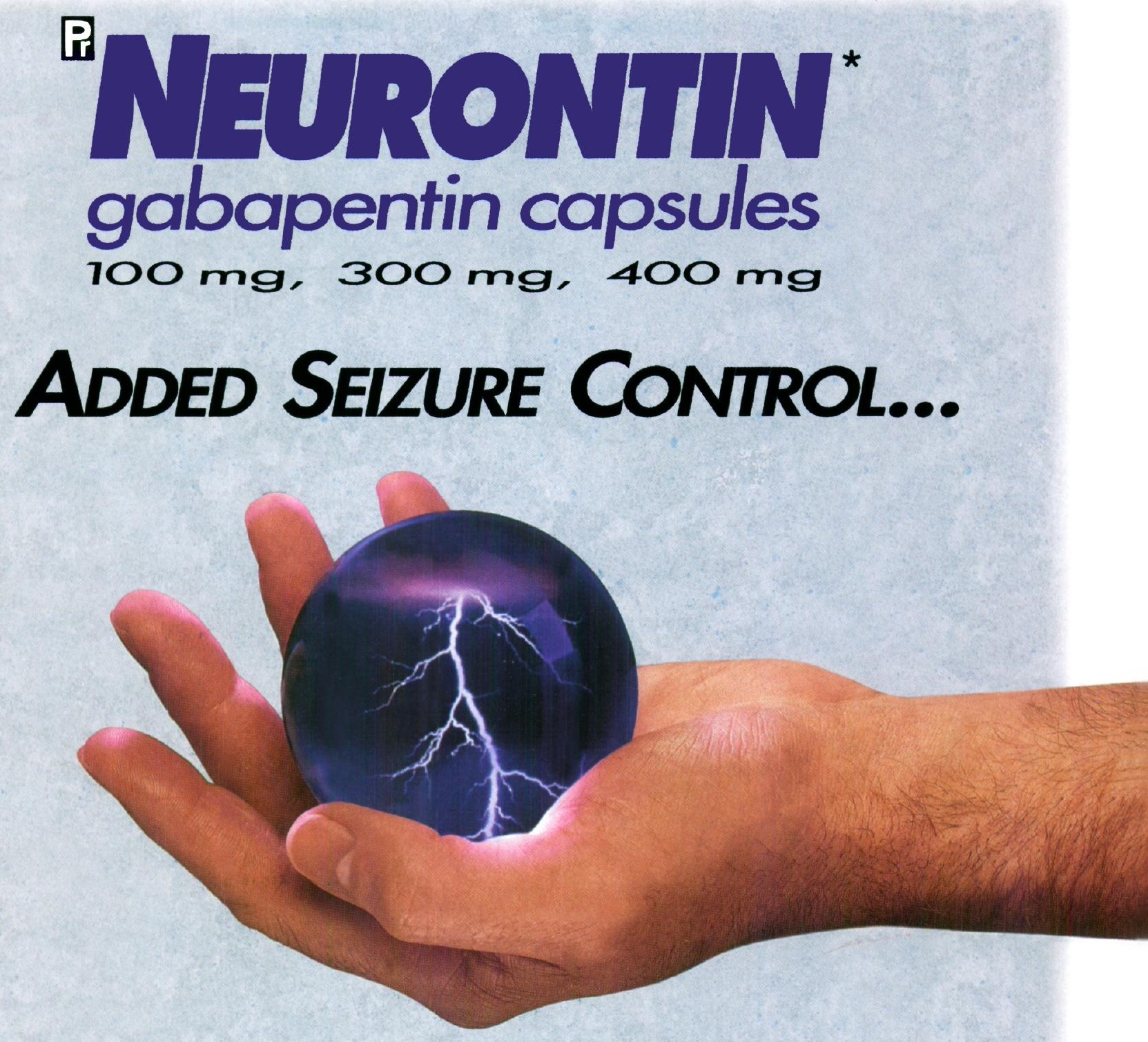

\section{... EASY TO HANDLE}

Neurontin is now available in Canada as adjunctive therapy to treat partial and secondarily generalized tonic-clonic seizures.
Unlike other adjunctive therapies, Neurontin has shown no pharmacokinetic interactions with standard anticonvulsants.'

Now combining therapies for added control is an easy choice with Neurontin. 

Nouveau Lamictal -

Traitement antiepleptique d'appoir

\section{La maîtrise d'un vaste éven un profil discret d'effets}

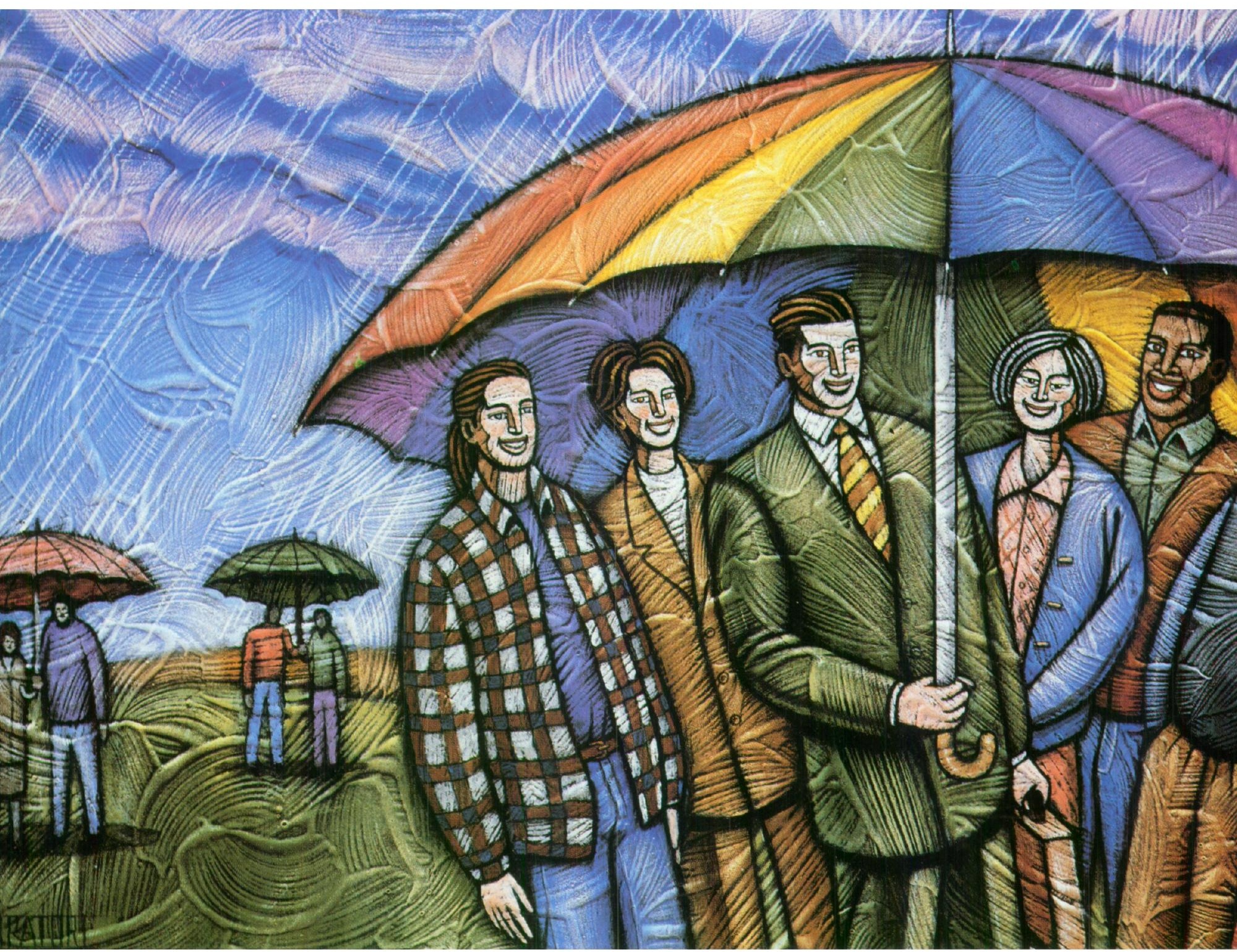

${ }^{\dagger}$ Taux d'abandon $(\geq 0,6 \%$ ) : étourdissements $2,4 \%$, céphalées $1,3 \%$, nausées $1,3 \%$, vision trouble $1,1 \%$, éruptions cutanées $1,1 \%$, diplopie $0,7 \%$, ataxie $0,6 \%$. En présence d'éruption cutanée inexpliquée, de fièvre, de symptômes pseudo-grippaux, ou de diminution de la maîtrise des crises, il faut surveiller les paramètres hépatiques, rénaux ou de coagulation. Voir dans la monographie du produit les recommandations chez les patients gériatriques et en cas d'atteinte rénale ou hépatique. De sérieux incidents cutanés peuvent être causés par un ajustement posologique initial rapide et l'emploi concomitant d'acide valprö́que.

łComme avec la plupart des autres antiépileptiques, avant de prescrire LAMICTAL, vérifier dans la monographie du produit les risques d'interaction médicamenteuse avec d'autres antiépileptiques.

Glaxo Wellcome 


\section{tail de types de crises avec secondaires sur le SNC}

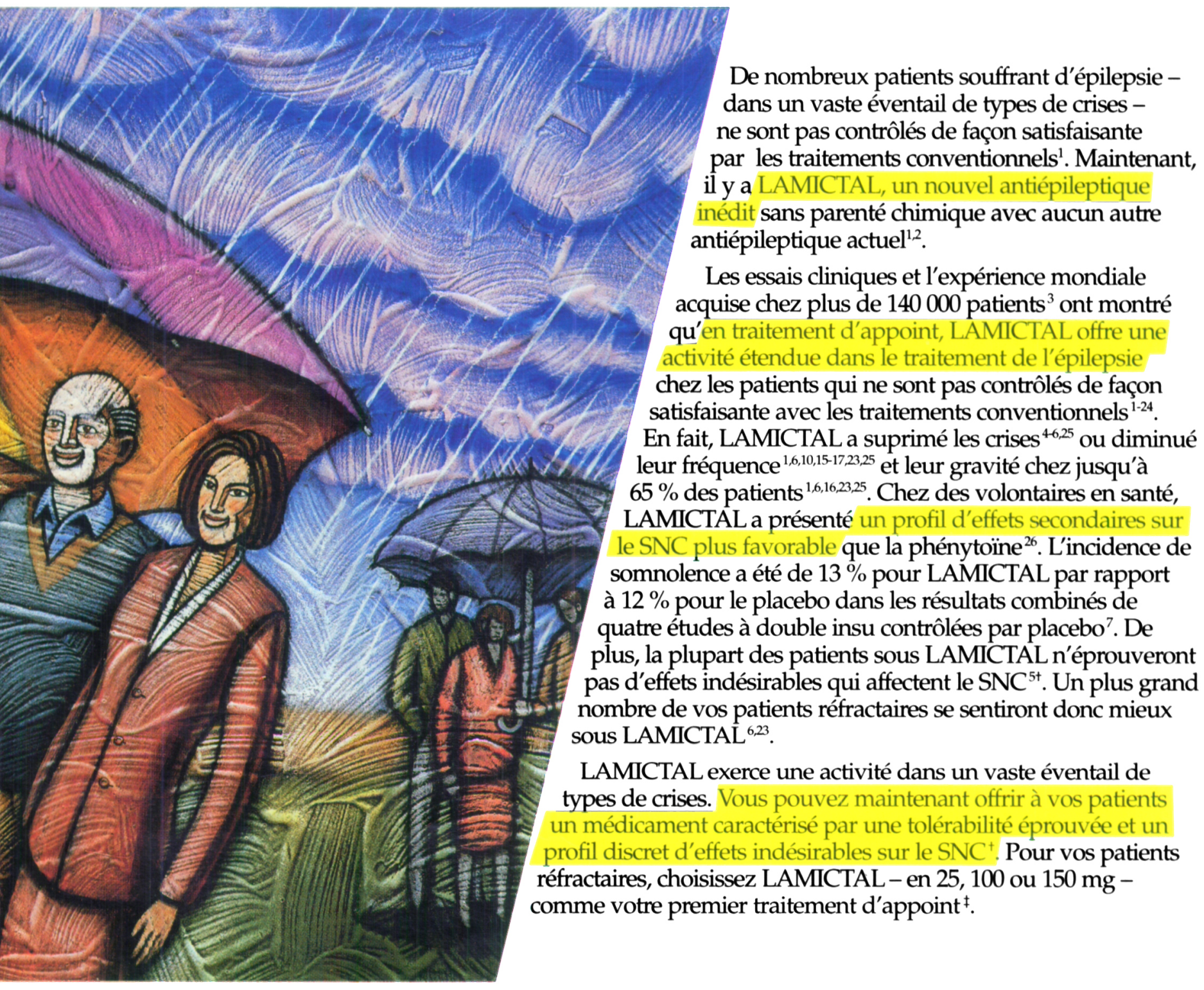

Nouveau!

Lamictal 


\section{Introducing \\ "BETASERON"}

The first treatment for relapsing/remitting multiple sclerosis

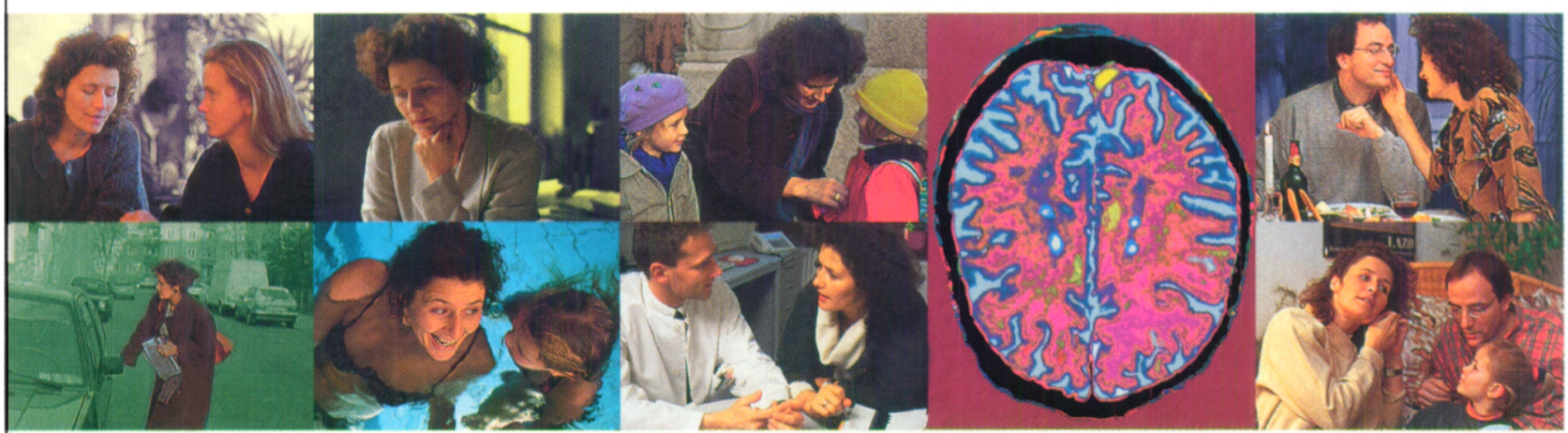

Clinical trials have shown that:

- The frequency of exacerbations was reduced

by approximately $30 \%^{1}$

- Moderate and severe exacerbations were reduced by $50 \%^{1}$

- Disease activity, as measured by MRI, was reduced significantly ${ }^{2}$

- There was a low incidence of serious side effects ${ }^{1}$

- Patient education about common side effects such as injection-site reactions and flu-like symptoms is key to compliance

Over 40,000 patients treated to date $\mathrm{e}^{3}$

\section{BETASERON \\ INTERFERON BETA-1b}

Maintaining Independence 


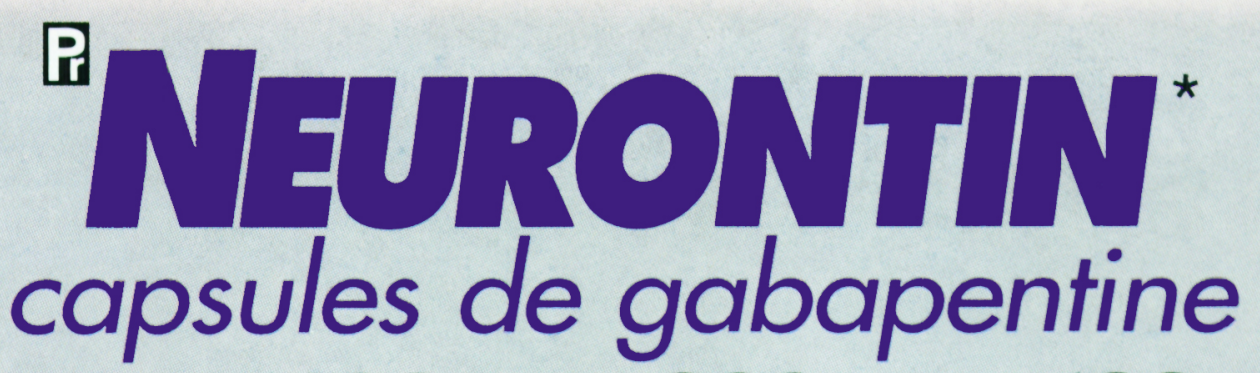

dosées à $100 \mathrm{mg}, 300 \mathrm{mg}, 400 \mathrm{mg}$

\section{POUR UNE MAÎTRISE SUPPLÉMENTAIRE DES CRISES D'EPILEPSIE...}

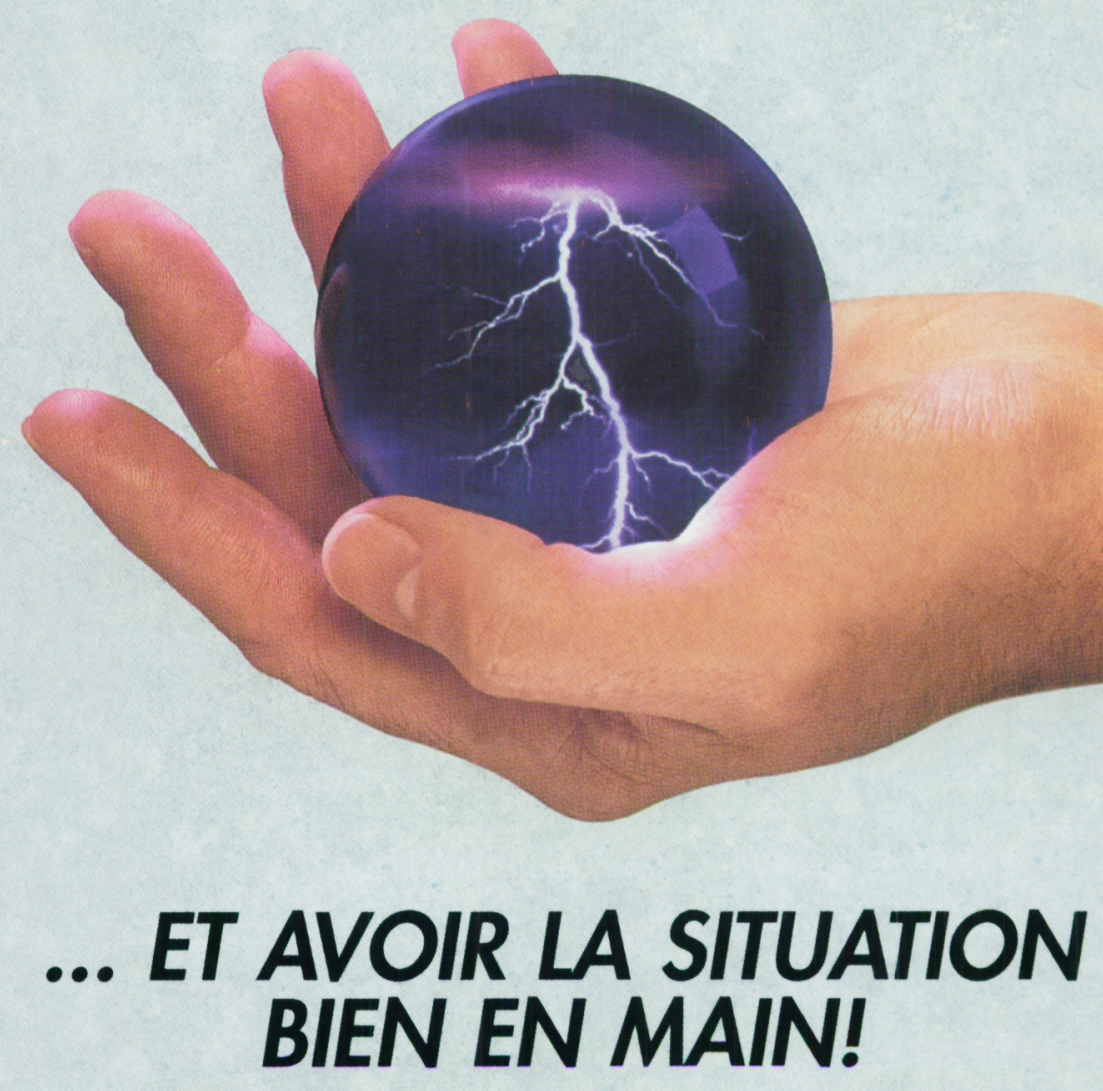

Neurontin est maintenant offert au Canada comme traitement adjuvant des crises partielles et tonico-cloniques secondairement généralisées.
Contrairement à ce qui se passe avec les autres traitements adjuvants, il n'y a pas $\mathrm{d}^{\prime}$ interaction pharmacocinétique entre Neurontin et les anticonvulsivants $\mathrm{d}^{\prime}$ usage courant ${ }^{+1}$.

Maintenant, avec Neurontin, la décision d'utiliser des traitements en association pour obtenir une maîtrise supplémentaire des crises est facile à prendre. 


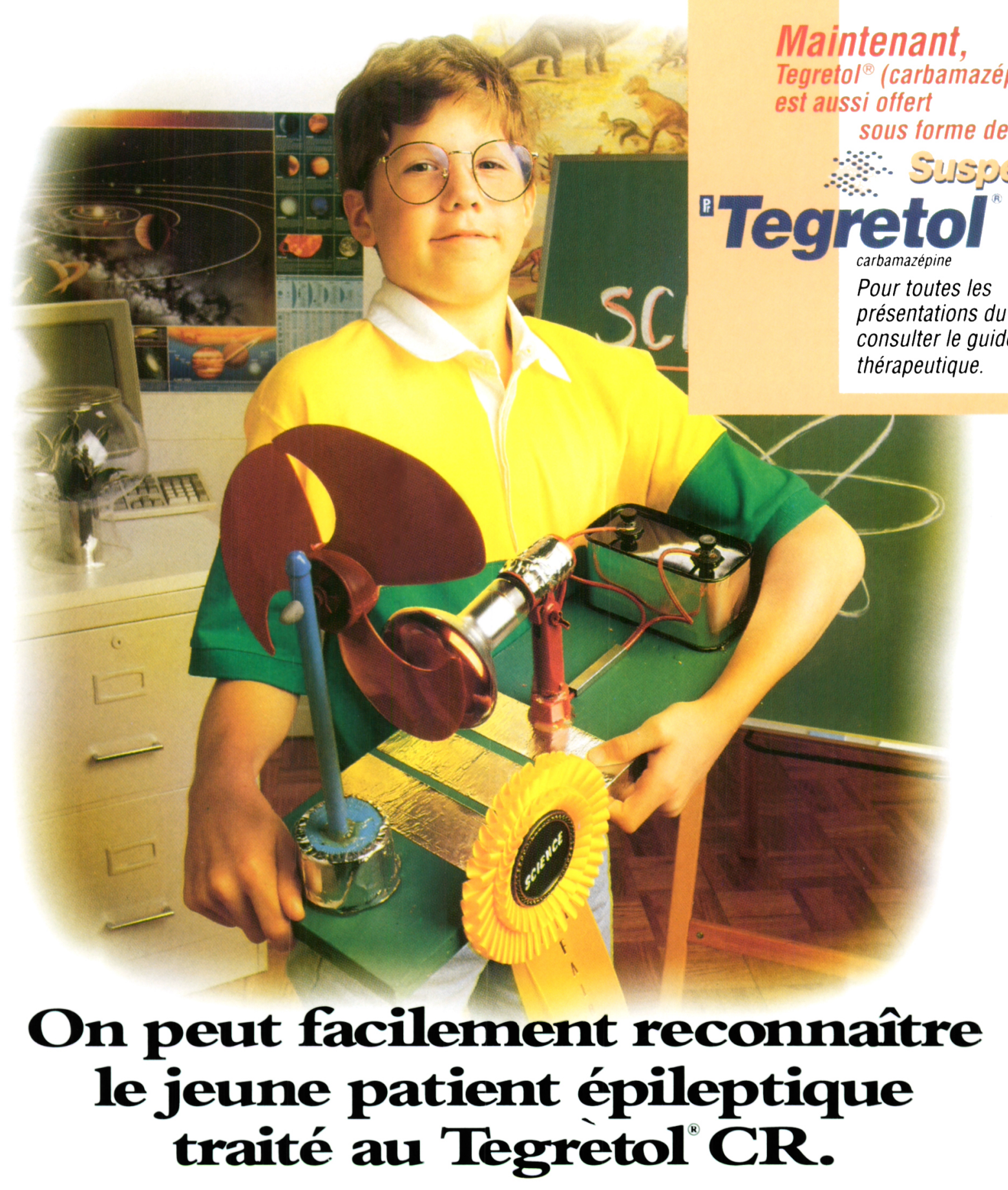

\section{Excellent contrôle des crises}

Q Tegretol $^{\circ} \mathrm{CR}$ (carbamazépine à libération contrôlée) maîtrise les crises chez de nombreux patients, causant peu d'impact sur la fonction cognitive ${ }^{1,2}$. Tegretol CR permet à de nombreux patients de penser clairement et de donner le meilleur d'eux-mêmes ${ }^{1.2}$.

\section{Taux sanguins uniformes}

Tegretol CR cause moins de "hauts et de bas" dans les taux sanguins que le Tegretol conventionnel. Les effets secondaires sont ainsi réduits et le modèle de fonction cognitive est plus stable.

\section{Posologie b.i.d. commode}

Lorsque vous instituez ou remplacez un traitement, pensez au Tegretol CR. Il est présenté en comprimés à $200 \mathrm{mg}$ et $400 \mathrm{mg}$ facilement divisibles pour une plus grande souplesse d'administration et améliorer

l'observance du patient.

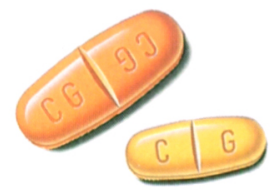

\section{TEGRETOL CR.}

Aide les épileptiques à réaliser leur plein potentiel. 
Reviewers, 1995

Adams, Harold

Alberts, Mark J.

Alexander, Michael

Ashby, Peter

Becker, Werner

Bedard, Paul

Benstead, Timothy J.

Bergeron, Catherine

Bernstein, Mark

Berry, Henry

Bilbao, John

Black, Sandra

Blume, Warren

Bolton, Charles

Bouchard, Jean-Pierre

Brooke, Michael $\mathrm{H}$.

Brown, John

Buchan, Alistair

Burnham, W.M.

Burns, Robert

Cairncross, Gregory

Camfield, Peter

Carpenter, Sterling

Chalk, Colin

Chertkow, Howard

Cote, Robert

Drake, James

Duke, Robert

Duquette, Pierre

Dyck, Peter

Eisen, Andrew

Elisevich, Kost V.

Fahn, Stanley

Feasby, Thomas

Fehlings, Michael

Fernie, Rosemary

Findlay, J. Max

Fletcher, William

Forsyth, Peter

Francis, Gordon

Fulton, Dorcas
Gauthier, Serge

Geggie, Peter H.

Gill, M. John

Guberman, Alan

Guha, Abhit

Guttman, Mark

Hagen, Neil

Hakim, Anthony

Halliday, William C.

Hansebout, Robert R.

Hassan, Mohamed N.

Heilman, Kenneth

Hoff, Julian

Hogan, David B.

Holness, Renn

Howse, D.C.N.

Humphreys, Peter

Hwang, Paul

Jankovic, Joe

Johnson, E.S.

Karlinsky, Harry J.

Karpati, George

Kirk, Andrew

Lang, Anthony E.

Laperriere, Normand J.

LeBlanc, Frank E.

Leblanc, Richard

Lee, Robert G.

MacDonald, David R.

Malkin, Mark

Maria, Bernard L.

Martin, Wayne

Maxner, C.E.

McGeer, Patrick

McLachlan, Richard S.

Mikulis, David

Muller, Paul

Munoz, David G.

Murray, Thomas J.

Myles, S. Terence

Norris, John W.
Noseworthy, John H.

Oger, Joel

Olanow, C. Warren

Peerless, Sidney J.

Picton, Terry

Power, Christopher

Pryse-Phillips, William

Rajput, A.H.

Riopelle, Richard

Rutka, James

Sadovnick, Dessa

Sarnat, Harvey B.

Schmidt, Brian

Schneiderman, Jacob $\mathrm{H}$.

Schondorf, Ronald

Seshia, S.

Seth, Kapil D.

Shapiro, Colin

Shelton, Paul

Shenouda, George

Sherwin, A.L.

Shorter, Edward

Shuaib, Ashfaq

Silver, Frank

Sima, Anders A.

Smith, Allan M.

Steinbok, Paul

Stewart, John D.

Stoessl, A. Jon

Strong, Michael

Stuss, Donald

Suchowersky, Oksana

Sutherland, Garnette

Teal, Phillip

Van Orman, Colin B.

Villemure, Jean-Guy

Wall, Michael

Weir, Bryce

Wong, C. Shun

Wong, Elaine

Zochodne, Douglas 